\title{
Experimentelle Untersuchungen über Autoimplantation von Nierengewebe ${ }^{1}$ ).
}

Von

A. Katz (Wien) und R. Gichtenstern (Wien).

(Mit Tafel IV und V.)

In einer früheren Versuchsreihe wurde von uns der Beweis erbracht, dass durch Schädigung der einen, im Organismus belassenen Niere die Produktion von Toxinen ausgelöst wird, welche eine elektive Wirkung auf die zweite Niere besitzen und zu schweren Allgemeinerscheinungen Anlass geben.

In diesen Versuchen wurde durch Unterbindung des Harnleiters ein allmählicher Schwund des Nierenparenchyms erzielt, und die bei der Einschmelzung des Gewebes sich bildenden Produkte sind es, welche die lokalen Läsionen an der zweiten Niere sowie die schweren Vergiftungserscheinungen der Versuchstiere hervorrufen. Die Toxinnatur der gebildeten Stoffe wird durch die gleichzeitig nachweisbare Entstehung spezifisch werdender Präzipitine bewiesen, wie auch von Ascoli bei ähnlicher Versuchsanordnung gefunden wurde. In Fortfübrung dieser Untersuchungen wurde in einer neuerlichen Versuchsreihe zu ermitteln gesucht, wie sich der tierische Organismus gegenüber der Implantation von Nierengewebe der gleichen Spezies verhält.

Auch Kapsenberg ${ }^{2}$ ) und $\mathrm{Pari}^{3}$ ) konstatierten das Auftreten und die Resorption von Autotoxinen beim Zerfall der eigenen Niere nach Unterbindung des Nierenstiels oder des Ureters, ersterer auch den Gehalt des Blutserums so vorbehandelter Tiere an Isozytotoxinen.

Die toxische Wirkung von Gewebsextrakten bei intravenöser Einspritzung wurde bereits mehrfach festgestellt. Aus den Organen tuberkulöser Meerschweinchen konnten Kraus und Volk ${ }^{4}$ ) mit

1) Die Versuche wurden unter gütiger Aufsicht des Herrn Prof. Dr. A. Kreidl im physiologischen Institute der Wiener Universität ausgeführt.

2) Zeitschr. f. Immunitätsforschung Bd. 12 S. 508, 513. 1912.

3) Zeitschr. f. Immanitätsforschung 1910.

4) Arch. ital. de Biol. t. 59 (2) p. 280. 
physiologischer Kochsalzlösung Stoffe extrahieren, die, in die Vene anderer Versuchstiere eingespritzt, rasch tödlich wirkten. Dass es sich hier nicht um Substanzen bandle, welche mit dem Grundleiden in Zusammenhang stehen, wies $\mathrm{Dold}^{\mathbf{1}}$ ) nach, der aus den Organen gesunder Tiere Extrakte gewann, die bei gleichartiger Einverleibung ebenfalls letal wirkten. Artgleiche Tiere wurden intensiver beeinflusst als die Versuchstiere einer fremden Spezies. Von D old wurde weiter die entgiftende Wirksamkeit des Blutserums festgestellt und gefunden, dass auch hier sich eine intensivere Wirksamkeit des artgleichen Serums feststellen lässt. Die intraperitoneale oder subkutane Einspritzung der Extrakte erwies sich als ungefährlich; selbst das Vierfache der bei intravenöser Infusion letalen Dosis war ungiftig. Über die Natur dieser Toxine spricht sich Dold des näheren nicht aus. Er erbringt nur den Beweis, dass es sich nicht um Seifen oder Lipoide handeln kann, dass weiter auch hämolytische Substanzen nicht als das totgebende Gift anzusehen sind. Bauer und Wusthoff ${ }^{2}$ ) heben den anaphylaktischen Charakter der Vergiftung durch Organextrakte besonders hervor. Der Sektionsbefund erbringt den Nachweis des Vorhandenseins von häufigen Thromben in den Venen und dem rechten Vorhof und veranlasst $L$ e $0 L_{0} e^{3}$ ), diese Versuche über Gewebstoxine mit früheren Versuchen von Conradi, Fuld und Boggs sowie den eigenen Experimenten ${ }^{4}$ ) in Zusammenhang zu bringen, welche fanden, dass in den Pressäften oder Extrakten der verschiedenen Organe ein Stoff vorhanden ist, der intravaskuläre Blutgerinnung bedingt (Kinase, Cytozym, Gewebskoagulum). Loeb's Versuche konnten in vitro die gerinnungserregende Wirkung der Gewebsextrakte auf Pepton- und Hirudinblut nachweisen und die Abschwächung der gerinnungserregenden Wirkung durch Blutserum feststellen. Diese Wirksamkeit des Blutserums war bis zu einem gewissen Grade spezifisch. Auch Blaizot ${ }^{5}$ ) führte die Giftigkeit der Organextrakte auf die Gegenwart von Thrombozym zurück. Diese Giftwirkung wird mit Vernichtung des Thrombozyms durch Zusatz von auf $56^{\circ}$ erhitztes Oxalatplasma, dem eine entsprechende Menge Kalk zugesetzt worden war, aufgehoben.

1) Zeitschr. f. Immunitätsforschung Bd. 10 S. 54.1911.

2) Deutsche med. Wochenschr. 1912 S. 894.

9) Zeitschr. f. Immunitätsforschung Bd. 12 S. 189. 1913.

4) Virchow's Arch. Bd. 201.

5) Compt. rend. Soc, de biol. t. 71 p. 534, 2. Dez. 1911. 
In Fortfübrung der Versuche intravenöser Einverleibung von Organemulsionen konnte Kapsenberg ${ }^{1}$ ) speziell für die Niere feststellen, dass intravenöse Injektion einer aus der einen Niere des Versuchstieres dargestellten Emulsion bereits in Mengen von $0,3^{\prime} \mathrm{g}$ pro Kilo Körpergewicht letal wirkt, während erst 2,0 g Nierengewebe, der Niere eines zweiten Kaninchens entstammend, den Tod herbeiführen.

Diese Versuche beweisen wohl, dass es sich nicht um embolische Erscheinungen handeln kann. Dagegen sprechen, wie auch Kapsenberg berichtet, die relative Unschädlichkeit von mit Lebersubstanz dargestellten Emulsionen wie die Feinheit der Emulsionierung an sich; auch der Symptomenkomplex nach Infusion der Nierensubstanz: Paralyse, Krämpfe, Atemstillstand, trotz Einleitung künstlicher Atmung. Der Sektionsbefund: rechter Herzventrikel in Diastole, linker in Systole, blutige Exsudate im Peritoneum und in den Lungen, spricht für eine akute Intoxikation.

In unseren Versuchen wurde, un die plötzliche, bei intravenöser Einspritzung stattfindende Überflutung des Organismus mit giftigen Stoffen hintanzuhalten, der Weg der Implantation von Organen oder Organteilen ins Peritoneum gewählt. Wird dafür Sorge getragen, dass die einzupflanzenden Gewebe ins grosse Netz eingehüllt werden, so ist deren Zerfall ein langsamer, die Resorption eine stetige, allmählich erfolgende. Der Eingriff, welcher zu dieser Gewebsimplantation notwendig wird, ist ein so geringfügiger, dass er an sich keinerlei nennenswerte Schädigungen nach sich ziehen kann: Durch einen kleinen Finschnitt in der Linea alba wird das Omentum vorgezogen und das in kleine Stückchen zerschnittene Organ in die gebildete Netztasche eingebracht; durch eine Tabaksbeutelnaht wird die Tasche verschlossen und in die Tiefe versenkt. Peritoneum und Bauchdecken werden hierauf sorgsam vernäht. Die Versucbe wurden an Katzen durchgeführt. Zur Implantation dienten die aseptisch entnommenen Nieren einer zweiten Katze. Dieselben wurden ibrer Kapsel entledigt, die Rinden- und Marksubstanz nach Entfernung des Nierenbeckens in kleine Partikelchen zerschnitten, mit physio. logischer, blutwarmer Kochsalzlösung gewaschen und in die gebildete Netztasche gelegt, diese hierauf verschlossen und versenkt.

Die Tiere erholen sich nach der Operation sehr raseh. An

1) Zeitschr. f. Immunitätsforschung Bd. 12 S. 508. 1912. 
dem ersten oder den zwei der Operation folgenden Tagen wird das Futter zurückgewiesen. Hierauf sind die Tiere ganz munter und nach glatter Verheilung der kleinen Bauchwunde von normalen nicht zu unterscheiden; trotzdem lässt aber eine genauere Untersuchung des Stoffwechsels sowohl als der Resistenz gegen Schädigungen erkennen, dass sich in ihrem Organismus Veränderungen abspielen, welche ihr physiologisches Gleichgewicht gestört haben. Die anatomische Untersuchung der Nieren in verschiedenen Intervallen nach Einpflanzung des Nierengewebes bringt die Bestätigung des Vorhandenseins markanter Organveränderungen.

Wie später ausführlicher erörtert wird, fanden wir anfangs Veränderungen des Nierengewebes ähnlich dem Bilde akuter hämorrhagischer Nephritis, in späterer Zeit Bilder, welche nephritisehen, in Ausheilung begriffenen Prozessen ähneln.

Die Beobachtung der im Stoffwechselkäfig gehaltenen Katzen ergab an dem der Operation folgenden Tage nicht selten Anurie; doch kann dies nicht mit Sicherheit auf den Eingriff bezogen werden, da wir sie auch sonst bei gesunden im Käfig gehaltenen Katzen beobachtet haben. Sie sind durch eine Polyurie am nächsten oder den nächsten Tagen ausgeglichen.

Das spezifische Gewicht, die Konzentration des Harns anzeigend, ist grossen Schwankungen unterworfen. Selbst dann, wenn durch absolute Milchdiät für gleichmässige Nahrungszufuhr gesorgt wird, zeigt die Zusammensetzung des Harns von Tag zu Tag sich ändernde, ganz regellose Schwankungen. Die gleichen, regellosen Schwankungen zeigt auch die Stickstoffausscheidung, trotz stets gleichbleibender Stickstoffeinfuhr in Form von Milch. Ein Stickstoffgleichgewicht war nicht zu erzielen. Die Stickstoffausfuhr gab oft Werte, welche die Einfuhr sehr wesentlich übersteigen. Diese Schwankungen, welche aus der Zufuhr in der Nahrung sich nicht erklären lassen, sind wohl als Beweis für die ungleichmässige Resorption des eingebrachten Materials, respektive als Folge der hierdureh bedingten Stoffwechselstörung heranzuziehen. Die zuweilen beobachtete starke Abmagerung trotz genügender Nahrungszufuhr - beweist, dass gesteigerter Zerfall im Verlaufe der Resorption statthat.

Die Kurve der Ammoniakausschejdung zeigt einen ebenso unregelmässigen Verlauf wie jene der Stickstoffzufuhr. Ein Parallelismus der beiden Kurven ist nicht zu konstatieren. Da die mikroskopische und chemische Untersuchung des implantierten Nierengewebes weitgehenden fettigen Zerfall aufweist, ist wohl das zeit- 
weise In-die-Höhe-gehen der Ammoniakausscheidung auf die schubweise erfolgende Resorption der gebildeten Fettsäuren zu beziehen.

Die Ausscheidung der Chloride zeigt analoge Schwankungen wie jene der früher genannten Stoffe. Bei der chlorarmen Milehnahrung sind diese Schwankungen nicht so ausgesprochen, die Kurve nähert sich der Einfuhr entsprechend mehr der Geraden: eine Stütze der Anschauung, dass die Schwankungen in der Ausfuhr von Stickstoff und Ammoniak nicht auf Rechnung unregelmässiger Nahrungsresorption, sondern infolge der durch Implantation bedingten Organveränderungen hervorgerufen werden.

(Siehe Tabelle I auf S. 420 und Tabelle II auf S. 421.)

Aus den Stoffwechseluntersuchungen lässt sich demnach der Schluss ableiten, dass bei den anseheinend ganz normalen Versuchstieren ein Stickstoffgleichgewicht nicht $z \mathfrak{u}$ erzielen ist, und dass abnorme Stoffwechselvorgänge als Folgen der Resorption des zerfallenden Organs sich eingestellt haben.

Die spezifische Beeinflussung der Nieren durch die beim Zerfall des implantierten Nierengewebes entstehenden Toxine wird durch die unmittelbar nach dem Eingriff auftretende, nicht unerhebliche Eiweissausscheidung mit dem charakteristischen, die Nierenreizung verratenden Sediment erwiesen. Die Eiweissmengen schwanken in weiteren Grenzen von Spuren bis zu 2\%. Der Gang der Albuminurie ist kein gleichmässiger. An einzelnen Tagen werden hohe Werte, an anderen nur geringe Mengen gefunden. Es liegt wohl nahe, anzunehmen, dass dieses schubweise Auftreten gesteigerter Eiweissmengen durch bald stärkere, bald schwächere Resorption toxischer Stoffe bedingt ist. Längere Zeit nach der Nierenimplantation ist die Albuminurie ganz oder bis auf ein unbedeutendes Minimum gesunken, und auch die Zylinder sind aus dem Sediment geschwunden.

Wird nun neuerlich eine Implantation von Nierengewebe vorgenommen, so geht die Eiweissausscheidung neuerlich in die Höhe: so schwand in einem Falle drei Wochen nach Eiubringen der Substanz zweier Nieren das Eiweiss vollständig aus dem Harn, trat bei neuerlicher Implantation der Substanz zweier Nieren wieder auf, sank dann wieder in wenigen Tagen auf Spuren, um nach einer dritten gleich grossen Dosis von Nierensubstanz neuerlich bis zu Werten von $0,1-0,26 \%$ anzusteigen und längere Zeit als nach der zweiten Operation anzuhalten. Auch hier sinkt die Albuminurie wieder auf quantitativ nicht bestimmbare Spuren ab. 


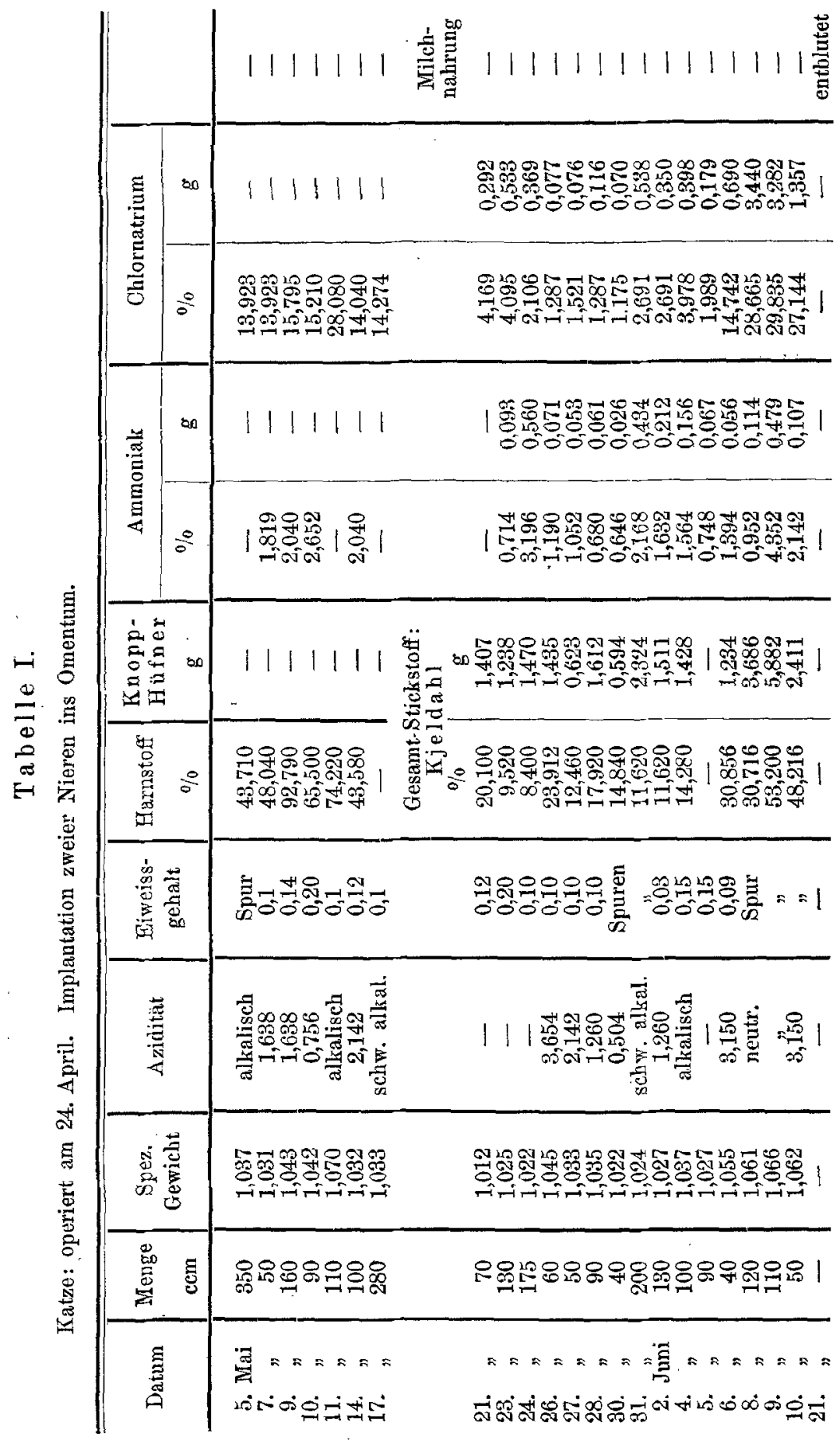


Exper. Untersuchungen über Autoimplantation von Nierengewebe.

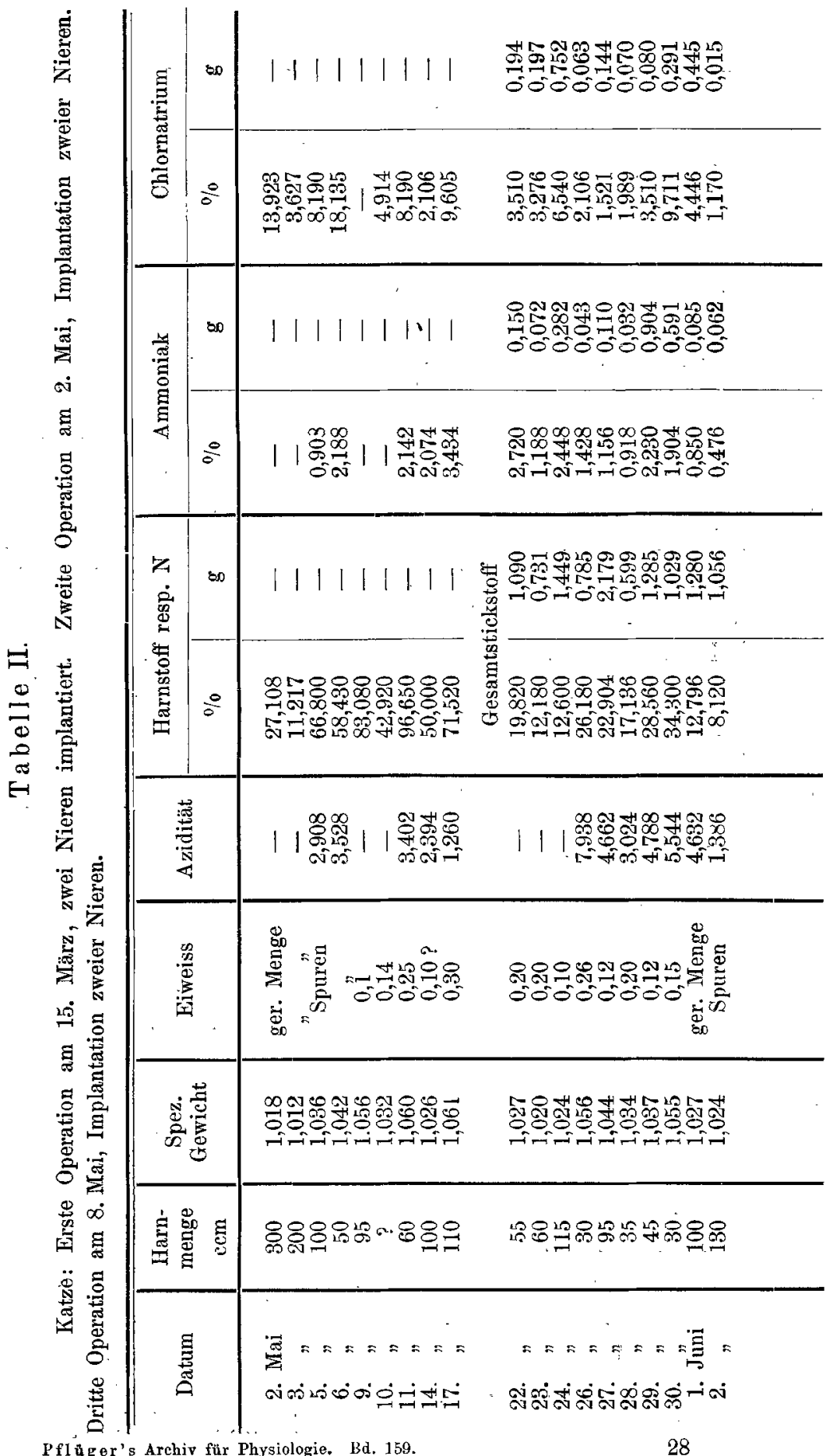

Pflüger's Archiv für Physiologie. Bd. 159. 
Aus dem Umstand, dass bei wiederholter Einbringung von Nierensubstanz der Organismus immer längere Zeit braucht, um der eingedrungenen Schädiguvg wieder Herr zu werden, lässt sich wohl schliessen, dass seine Widerstandsfähigkeit abgenommen hat, ohne aber, wie ja das endliche Verschwinden der Albuminurie beweist, ganz aufgehoben zu sein. Die Wiederholung der Einbringung von Nierengewebe scheint dem Körper eine gewisse Inmunität gegen die Toxine zu verleihen, während die einmalige Überflutung mit grossen Mengen sich bildenden Gifte nicht ertragen wird. Bei dieser Versuchsreihe liegen daher analoge Verhältnisse vor wie bei intravenöser Injektion von Organextrakten, die, wie erwähnt, rasch zum Tode der Versuchstiere führen. Das Blutserum nicht immunisierter Tiere scheint zu wenig Antitoxin zu enthalten, um seine von Dold erwiesene Schutzwirkung in ausreichendem Masse zu entfalten.

Wird bei einer Katze die Substanz von vier Nieren auf einmal in das Peritoneum eingebracht, so geht das Tier rasch ein. Die Niere vermag offenbar die übergrosse Belastung durch die sich rapid entwickelnden Toxine nicht auszuhalten und sondert bis zu dem nach 2-3 Tagen erfolgenden Torle keinen Harn mehr ab. Die Sektion zeigte ausser akut entzündlichen Veränderungen des Nierenparenchyms und einer Milzschwellung keine pathologischen Veränderungen im Organismus und nie Zeichen irgendeiner durch den operativen Eingriff bedingten Infektion.

Als weitere Stütze der elektiven Wirkung der bei dem Zerfall der eingebrachten Nierensubstanz entstandenen Toxine auf die Nieren des Versuchstieres dienen die Beobachtungen an Katzen, bei welchen durch Uraneinspritzung die Nieren geschädigt wurden. Wenn diesen Tieren die sonst nicht letale Dosis von zwei Nieren implantiert wurde, so gingen sie unter urämischen Erscheinungen nach 2-3 Tagen zugrunde, während sonst die Uranwirkung in einigen Tagen nach der Injektion abklingt. Aber auch dann, wenn Abnahme der Eiweissausscheidung darauf hinweist, dass die Uranwirkung in Abnahme begriffen ist, vermag die geschwächte Niere nicht mehr genügenden Widerstand zu leisten. Wurde bei einer Katze, welche vor vier Tagen $0,01 \mathrm{~g}$ Urannitrat subkutan erhalten hatte und bei der die Eiweissausscheidung von $0,3 \%$ auf sehr geringe Mengen gesunken und bei der auch die Zuckerausseheidung in Abnahme begriffen war, die Substanz zweier Nieren implantiert, so ging das Tier nach ca. 36 Stunden unter Versagen der Nierentätigkeit und beinahe kompletter Anurie zugrunde. 
Exper. Untersuchungen über Autoimplantation von Nierengewebe. 423

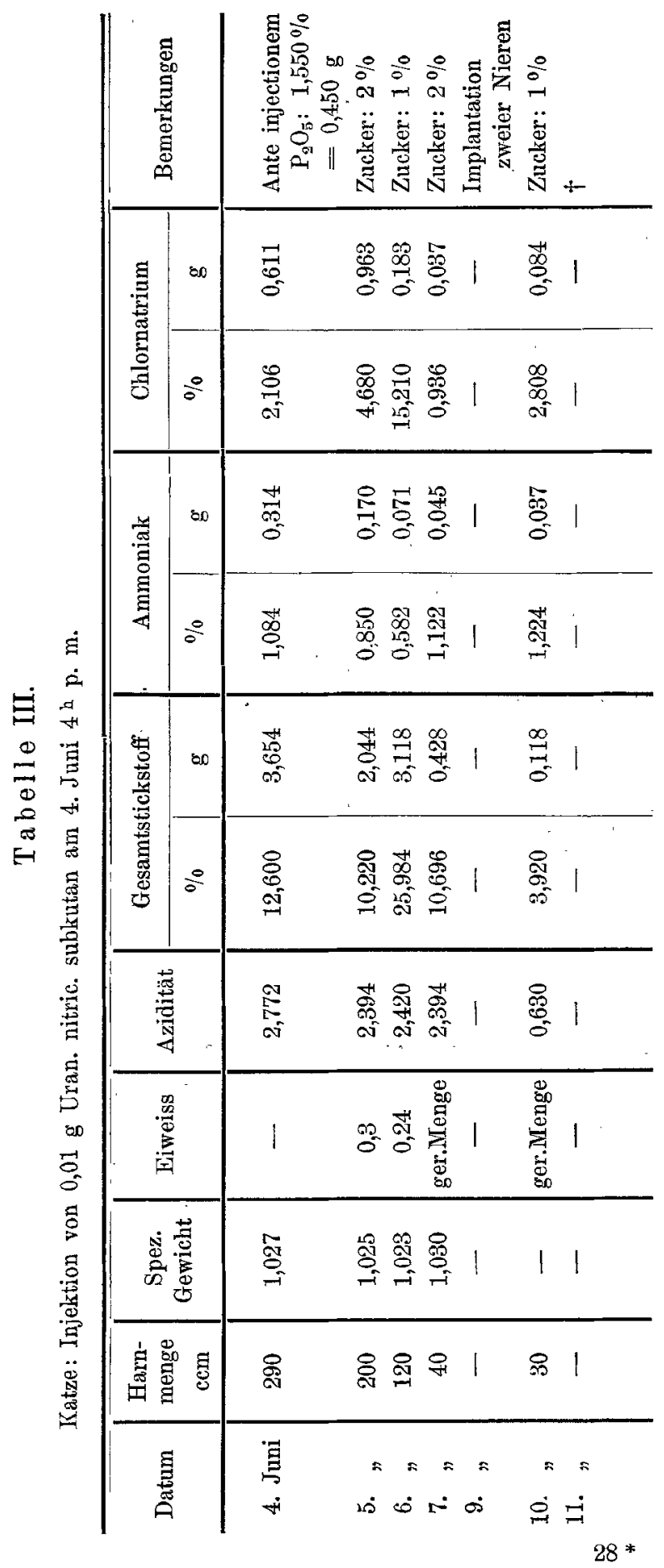


Die histologische Untersuchung der Nieren derjenigen Versuchstiere, die nach Implantation grosser Mengen von Nierensubstanz rasch zugrunde gegangen waren, zeigte das Bild einer hämorrhagischen Nephritis (Fig. 3): die Gefässe waren trotzend mit Blut gefüllt, stellenweise fanden sich in die Kapselräume ausgetretene rote Blutkörperchen, stellenweise seröses Exsudat. Die zu einem solchen Gebiete gehörenden Tubuli contorti zeigten das Bild schwerster Schädigung, hyaline und granulierte Zylinder, stellenweise auch beginnende Verkalkung.

Die Untersuchung jener Nieren, die Versuchstieren entstammten, welche mehrfache Implantationen gut überstanden hatten und bei anscheinend normalem Verhalten getötet worden waren, zeigten Schädigungen an den Glomerulis, in den peripheren Abschnitten, Veränderungen vom Typus junger Regeneration, die Marksubstanz nicht wesentlich verändert, also das Bild einer in Ausheilung begriffenen Nephritis (Fig. 2).

In den implantierten Stücken, die vier Wochen nach dem Eingriff der Untersuchung zugeführt wurden, zeigten sich an verschiedenen Stellen Vorstadien beginnender Verkalkung an den abgestorbenen Zellen der Harnkanälchen, weiter zellreiches Fettgewebe mit kleinsten Resten von Marksubstanz.

Auf Grund der durchgeführten Versuche lässt sich annehmen:

1. Die Resorption von zerfallendem Nierengewebe hat eine schwere, wenn auch reparable Stoffwechselstörung und schwere, einer Regression zugängliche anatomische Veränderung der Niere zur Folge.

2. Der in Heilung begriffene Prozess kann durch neuerliche Zufuhr von zerfallendem Nierengewebe im gleichen Sinne wieder angefacht werden.

3. Die Wirkung des resorbierten Materials ist in elektiver Weise auf die Niere gerichtet, und bei geschädigter kranker Niere kann keine Restitution stattfinden. 
Exper. Untersuchungen über Autoimplantation von Nierengewebe. 425

\section{Tafelerklärung.}

\section{Tafel IV.}

Fig. 1. Akute Nephritis. Kapselraum erweitert mit Exsudat. Leukocyten. Tubuli noch nicht geschädigt.

Fig. 2. Subakute Glomerulitis, zum Teil interstitiell sich ausbreitend, Erweiterung der Bowman' schen Kapseln mit reichlichem Exsudat mit Rundzellen; Tubuli im Zustande parenchymatöser Degeneration. Unter der Nierenkapsel Verkalkungsherde.

\section{Tafel V.}

Fig. 3. Akute hämorrhagische Nephritis. Blutiges Exsudat und Rundzellen im Kapselraum sowie im interstitiellen Bindegewebe (ungefähr das Bild wie bei Scharlachnephritis). 